\title{
Publisher Correction: Questioning life
}

Guy Ben-Ary

Correction to: Nature Nanotechnology https://doi.org/10.1038/s41565-019-0444-8, published online 7 May 2019.

In the version of this Feature originally published, an older standfirst was used by mistake; it should have read 'Art-based absurd scenarios serve as a vector to challenge perceptions of emergent biotechnologies.' The author's affiliation was missing 'School of Human Sciences'; it should have read 'SymbioticA, Centre of Excellence for Biological Arts, School of Human Sciences, University of Western Australia, Perth, Western Australia, Australia. The author also wishes to add, after 'For example, cellF is a collaborative project', the following text '(with artists Nathan Thompson, Andrew Fitch and Darren Moore, and scientists Stuart Hodgetts, Mike Edel and Douglas Bakkum)'.

Published online: 15 May 2019

https://doi.org/10.1038/s41565-019-0473-3

\section{Publisher Correction: The poetry of materials}

Thomas Duggan and S. J. Fowler

Correction to: Nature Nanotechnology https://doi.org/10.1038/s41565-019-0450-x, published online 7 May 2019.

In the version of this Feature originally published, the authors' websites, www.thomasdugganstudio.com and www.stevenjfowler.com, were missing from the affiliations. This has now been corrected.

Published online: 17 May 2019

https://doi.org/10.1038/s41565-019-0474-2 